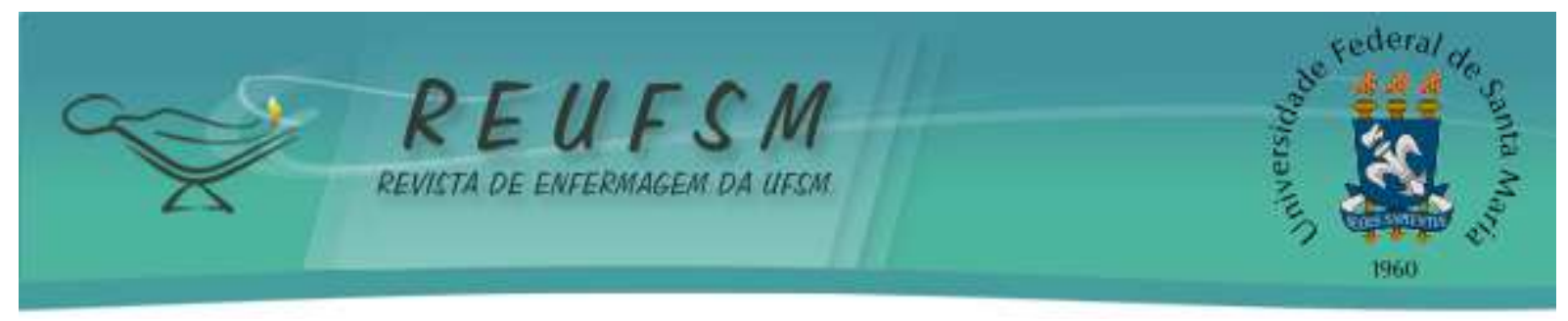

\title{
AMBIENTE DE TRABALHO DO ENFERMEIRO EM UNIDADES DE CLÍNICA MÉDICA E CIRÚRGICA
}

\section{WORK ENVIRONMENT OF THE NURSE IN CLINICAL AND SURGICAL UNITS \\ AMBIENTE DE TRABAJO DEL ENFERMERO EN UNIDADES DE CLÍNICA MÉDICA Y QUIRÚRGICA}

\author{
José Luís Guedes dos Santos ${ }^{1}$ \\ Fernando Henrique Antunes Menegon ${ }^{2}$ \\ Dulcinéia Ghizoni Schneider ${ }^{3}$ \\ Giovana Dorneles Callegaro Higashi ${ }^{4}$ \\ Fernanda Hannah da Silva Copelli ${ }^{5}$ \\ Alacoque Lorenzini Erdmann ${ }^{6}$
}

Doi: $10.5902 / 2179769229506$

RESUMO: Objetivo: analisar o ambiente de trabalho do enfermeiro em unidades de clínica médica e cirúrgica. Método: pesquisa de método misto, com estratégia triangulação concomitante de dados. Os dados quantitativos foram coletados com 27 enfermeiros de um Hospital Universitário do Sul do Brasil, por meio do Brazilian Nursing Work Index Revised $(B-N W I-R)$ e submetidos à análise estatística descritiva. Os dados qualitativos foram obtidos a partir de 14 entrevistas e tratados mediante análise temática. Resultados: os enfermeiros possuem autonomia, têm um bom relacionamento com os médicos e suporte organizacional para o trabalho de enfermagem. No entanto, o controle sobre o ambiente é prejudicado pela falta de pessoal de enfermagem e suporte dos serviços de apoio. Conclusão: o estudo evidenciou aspectos que podem melhorar o ambiente de prática de enfermeiros de unidades de clínica médica-cirúrgica e contribuir para segurança e qualidade do cuidado de enfermagem.

Descritores: Ambiente de trabalho; Organização e administração; Enfermagem; Pesquisa em administração de enfermagem; Hospitais públicos.

ABSTRACT: Aim: to analyze the work environment of the nurse in medical and surgical clinic units. Method: mixed method research, with concomitant triangulation of data strategy. The quantitative data were collected with 27 nurses of a University Hospital of the South of Brazil, through the Brazilian Nursing Work Index Revised (B-NWI-R) and submitted to descriptive statistical analysis. Qualitative data were obtained from 14 interviews and treated using thematic analysis. Results: nurses have autonomy, a good relationship with physicians

\footnotetext{
1 Enfermeiro. Doutor em Enfermagem. Professor Adjunto do Departamento de Enfermagem da Universidade Federal de Santa Catarina. Florianópolis, Santa Catarina, Brasil. E-mail: joseenfermagem@gmail.com

${ }^{2}$ Enfermeiro. Mestrando em Enfermagem do Programa de Pós-Graduação em Enfermagem da Universidade Federal de Santa Catarina. Florianópolis, Santa Catarina, Brasil. E-mail: fernandomenegon01@ gmail.com

3 Enfermeira. Doutora em Enfermagem. Professor Adjunto do Departamento de Enfermagem da Universidade Federal de Santa Catarina. Florianópolis, Santa Catarina, Brasil. E-mail: dulcineiags@gmail.com

${ }^{4}$ Enfermeira. Doutora em Enfermagem. Universidade Federal de Santa Catarina. Florianópolis, Santa Catarina, Brasil. E-mail: gio.enfermagem@gmail.com

${ }^{5}$ Enfermeira. Mestre em Enfermagem. Doutoranda em Enfermagem do Programa de Pós-Graduação em Enfermagem da Universidade Federal de Santa Catarina. Florianópolis, Santa Catarina, Brasil. E-mail: fernandacopelli@hotmail.com

6 Enfermeira. Doutora em Enfermagem. Professora Titular do Departamento de Enfermagem da Universidade Federal de Santa Catarina. Florianópolis, Santa Catarina, Brasil. E-mail: alacoque.erdmann@ufsc.br
} 


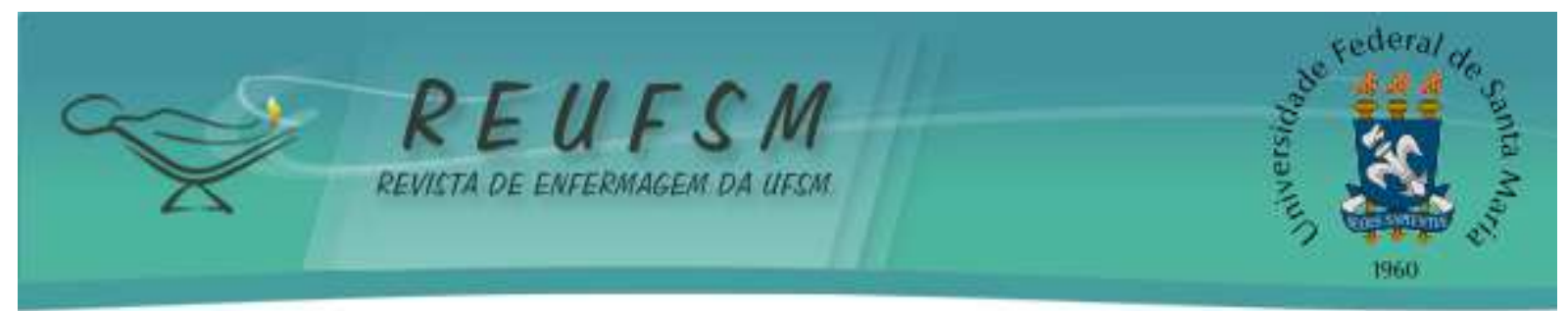

and an organizational support for nursing work. However, control over the environment is hampered by the lack of nursing staff and the assistance of support services. Conclusion: the study showed aspects that can improve the practice environment of nurses from medicalsurgical clinic units and contribute for the safety and quality of nursing care.

Descriptors: Working environment; Organization and administration; Nursing; Nursing administration research; Hospitals public.

RESUMEN: Objetivo: analizar el ambiente de trabajo del enfermero en unidades de clínica médica y quirúrgica. Método: investigación de metodología mixta, con estrategia triangulación concomitante de datos. Los datos cuantitativos fueron recolectados con 27 enfermeros de un Hospital Universitario del Sur de Brasil, a través del Brazilian Nursing Work Index Revised (B-NWI-R) y analizados por medio de estadística descriptiva. Los datos cualitativos fueron obtenidos por medio de 14 entrevistas y tratados con análisis temático. Resultados: los enfermeros poseen autonomía, tienen una buena relación con los médicos y soporte organizacional para el trabajo de enfermería. Sin embargo, el control sobre el ambiente es perjudicado por la falta de personal de enfermería y soporte de los servicios de apoyo. Conclusión: el estudio evidenció aspectos que pueden mejorar el ambiente de la práctica de enfermeros en unidades de clínica médico-quirúrgica y contribuir con la seguridad y calidad del cuidado de enfermería.

Descriptores: Ambiente de trabajo; Organización y administración; Enfermería; Investigación en administración de enfermería; Hospitales públicos.

\section{INTRODUÇÃO}

No Brasil, existem 493.149 leitos de internação distribuídos entre 6.706 hospitais. Desse total de leitos, 227.235 destinam-se à cirurgia e clínica geral, o que corresponde a $46 \%$ dos leitos hospitalares. ${ }^{1}$ Assim, constata-se que as unidades de internação clínica e cirúrgica são os locais onde a maioria das pessoas com necessidades de saúde que requerem hospitalização é atendida, configurando-se como espaços privilegiados de cuidado direto em uma instituição hospitalar. Consequentemente, concentram grande número de profissionais de enfermagem. ${ }^{2}$

O ambiente hospitalar dos setores de clínica médica e cirúrgica compõe-se de espaços físicos com leitos e demais equipamentos para a atenção à saúde às pessoas ali internadas, insumos e logística para a prática de cuidados, conforto e necessidades corporais e sociais, sob a atenção dos trabalhadores da saúde e de serviços complementares, cada um atuando na sua especificidade de cuidado, com a finalidade de restabelecer a saúde dos pacientes. Nesse contexto, o trabalho do enfermeiro abrange o atendimento às necessidades de cuidado de enfermagem dos pacientes que se concretiza nas dimensões clínica, educativa e gerencial e em abrangência múltipla, do micro ao macro ambiente/sistemas e do mais individual e privativo ao mais coletivo e participativo. Além disso, também envolve a provisão e previsão dos materiais 


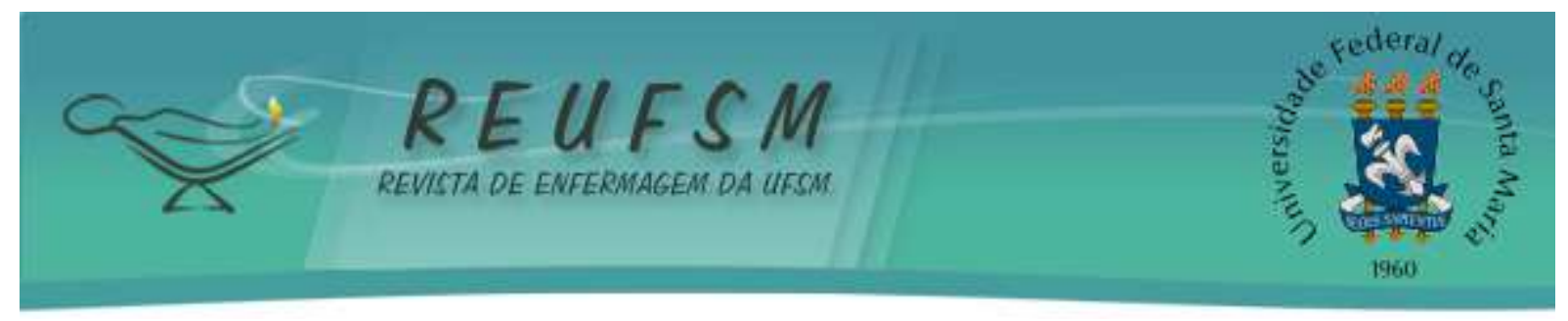

assistenciais necessários e a articulação do trabalho dos diversos profissionais da equipe de saúde de modo que o cuidado possa ser realizado com qualidade e segurança aos pacientes. ${ }^{2-3}$

Desse modo, para que o enfermeiro desenvolva suas atribuições com eficiência e qualidade é importante que as características do ambiente de trabalho sejam favoráveis à sua prática profissional. As condições físicas e organizacionais para o desempenho que se julguem adequados da prática profissional equivalem ao ambiente de trabalho do enfermeiro. Esse ambiente de trabalho compreende elementos como o modelo de gestão adotado pela instituição, a infraestrutura, recursos financeiros, cultura organizacional e o desenvolvimento da assistência. ${ }^{4}$

Inúmeras características podem influenciar o ambiente de trabalho e a prática profissional de enfermagem, permeando a liderança e autonomia na prática clínica e gerencial do enfermeiro. Consequentemente, ambientes de trabalho com características positivas possibilitam maior satisfação profissional dos enfermeiros e melhoria da qualidade assistencial no âmbito hospitalar. ${ }^{5-6}$

Apesar da influência do ambiente de trabalho na prática do enfermeiro, a produção científica brasileira sobre esse tema ainda é incipiente, sendo que as produções existentes concentram-se na região Sudeste e no âmbito de Unidades de Terapia Intensiva. ${ }^{7-9}$ Assim, na consulta a algumas das principais bibliotecas e bases de dados online, como Scientific Electronic Library Online (SciELO), Base de Dados em Enfermagem (BDENF), Literatura Latino-Americana em Ciências da Saúde (LILACS) e National Library of Medicine (PubMed), não foi identificada nenhuma publicação científica nacional cujo foco tenha sido a análise do ambiente de trabalho do enfermeiro em unidades clínica médica e cirúrgica.

Assim, este estudo teve como questão de pesquisa: Como se configura o ambiente de trabalho do enfermeiro em unidades de clínica médica e cirúrgica?

O objetivo da pesquisa foi analisar o ambiente de trabalho do enfermeiro em unidades de clínica médica e cirúrgica.

\section{MÉTODO}

Pesquisa de método misto, combinando abordagem qualitativa e quantitativa, por meio da estratégia triangulação concomitante de dados (QUAN + QUAL). Nesse tipo de estudo, os dados quantitativos e qualitativos são coletados concomitantemente e depois comparados com o objetivo de determinar convergências, diferenças e combinações entre eles. ${ }^{10}$ 


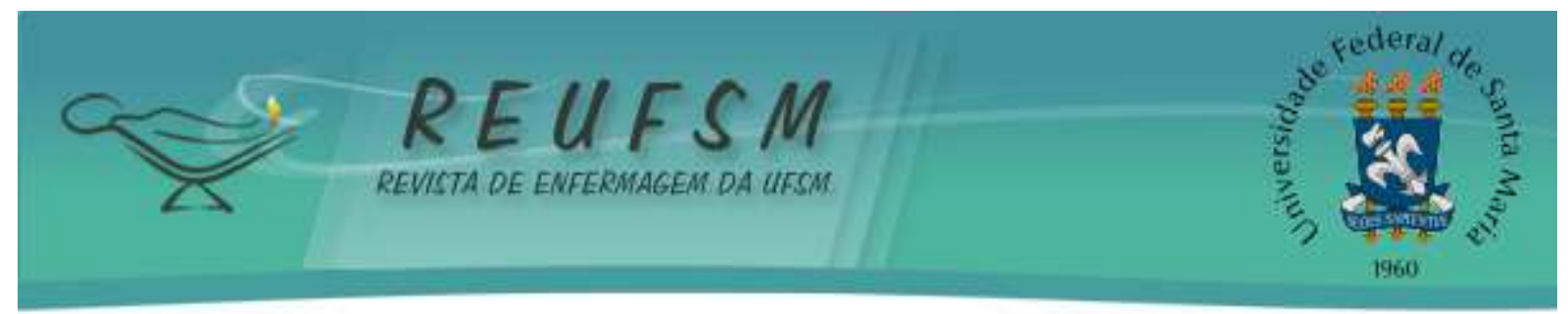

O local do estudo foram as cinco unidades de clínica médica e clínica cirúrgica de um hospital universitário do Sul do Brasil. A coleta dos dados foi realizada no período de outubro de 2012 a março de 2013, por estudantes de graduação e pós-graduação em enfermagem, capacitados por meio de encontros com os pesquisadores para discussão do tema de pesquisa e dos procedimentos metodológicos para coleta de dados, tanto antes quanto durante o desenvolvimento da pesquisa.

$\mathrm{Na}$ ocasião do estudo, as unidades de clínica médica e clínica cirúrgica do hospital contavam com 40 enfermeiros, o que correspondeu à população-alvo da pesquisa. Como critérios de inclusão, foram estabelecidos o exercício de atividades assistenciais e tempo de experiência igual ou maior que três meses no atual local de trabalho. Foram excluídos dois enfermeiros ausentes por motivo de férias ou licença de qualquer natureza. Dos 38 enfermeiros aptos a participar da pesquisa, sete não devolveram os instrumentos e quatro recusaram-se a participar da pesquisa. Dessa forma, a amostra investigada correspondeu a 27 enfermeiros.

Os dados quantitativos foram coletados por meio de uma ficha de caracterização sociodemográfica e o instrumento Brazilian Nursing Work Index - Revised (B-NWI-R). O BNWI-R foi traduzido, adaptado e validado para uso no Brasil e mensura características do ambiente de trabalho favoráveis à prática profissional do enfermeiro. ${ }^{11}$ Foram considerados os 15 itens do instrumento que compõem as quatro subescalas: autonomia (cinco itens), controle sobre o ambiente (sete itens), relações entre médicos e enfermeiros (três itens) e suporte organizacional (dez itens derivados das três primeiras subescalas). ${ }^{9,11}$

A escala de medida do B-NWI-R é do tipo Likert, cuja pontuação varia entre um a quatro pontos. $\mathrm{O}$ participante é solicitado a responder se concorda ou não com a afirmativa "esse fator está presente no meu trabalho diário", a partir das opções: concordo totalmente (um ponto), concordo parcialmente (dois pontos), discordo parcialmente (três pontos) e discordo totalmente (quatro pontos), ou seja, quanto menor a pontuação, maior a presença de atributos favoráveis. Valores abaixo de 2,5 representam ambientes favoráveis à prática profissional e médias acima de 2,5 pontos indicam ambientes desfavoráveis. ${ }^{9,11}$

Os dados quantitativos foram analisados no programa Statistical Package for the Social Sciences (SPSS), versão 19.0. Para apresentar os dados, utilizou-se estatística descritiva com cálculo de medidas de posição e dispersão (média, mediana, desvio-padrão, valores mínimo e 


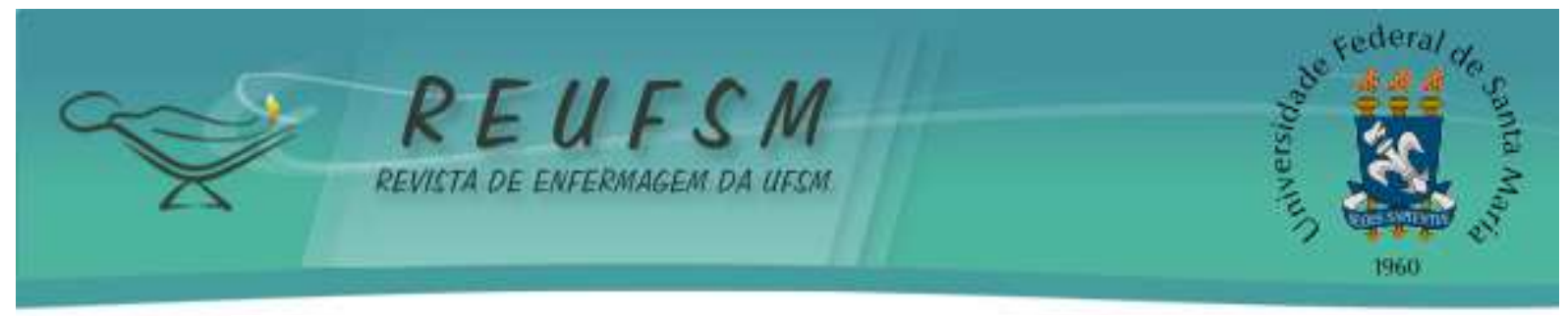

máximo). O coeficiente Alpha de Cronbach foi utilizado para avaliação da confiabilidade da escala global e suas dimensões. O valor do alfa de Cronbach geral foi de 0,790 .

Os dados qualitativos foram coletados por meio de entrevistas semiestruturadas com 14 enfermeiros que participaram da etapa quantitativa do estudo. Esse número de participantes foi definido a partir do critério de saturação dos dados, ou seja, a coleta de dados foi interrompida quando não se constataram mais novos elementos para subsidiar a teorização do estudo e os dados coletados foram considerados suficientes para responder o objetivo da pesquisa. ${ }^{12}$ As entrevistas foram audiogravadas, tiveram duração média de 20 minutos e continham questões sobre aspectos que contribuíam e/ou dificultavam a prática do enfermeiro nas unidades de clínica médica e cirúrgica. Cada participante foi entrevistado individualmente no seu local de trabalho ou outro ambiente da sua escolha.

Para análise dos dados, adotou-se a técnica de análise temática. Inicialmente, procedeu-se à pré-análise e exploração do material por meio de leitura exaustiva e compreensiva. Na sequência, realizou-se a exploração do material e tratamento dos resultados, inferência e interpretação. ${ }^{13}$ As subescalas do B-NWI-R foram utilizadas como categorias pré-determinadas para a organização e descrição dos resultados. Os depoimentos dos entrevistados foram identificados por códigos compostos pela letra "E" de enfermeiro e um número atribuído conforme ordem das entrevistas: E1, E2,..., E14. A Figura 1 apresenta uma síntese do desenho misto da pesquisa.

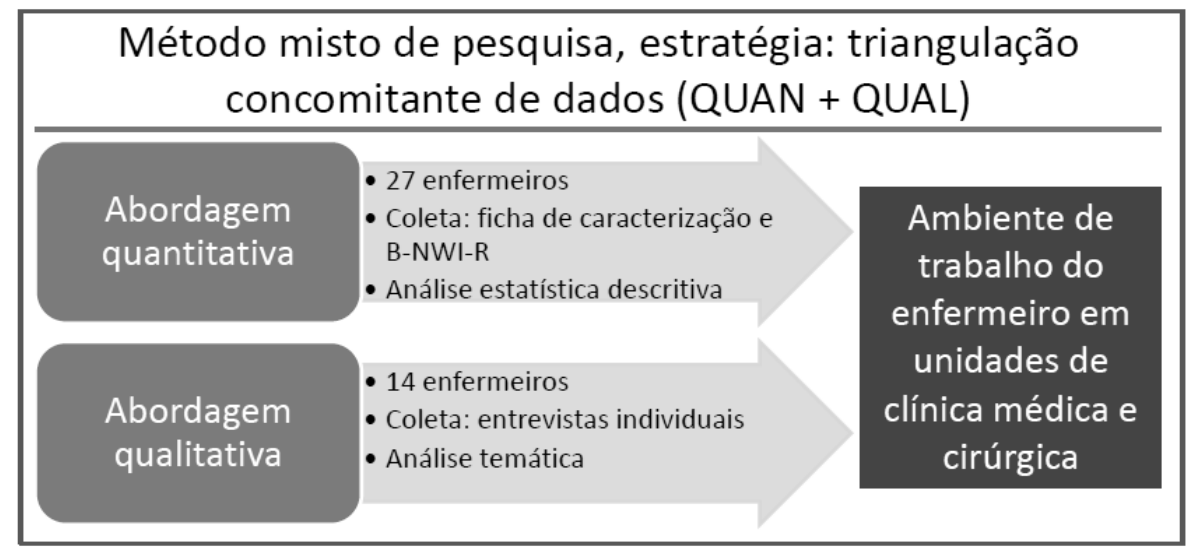

Figura 1 - Desenho da pesquisa

A pesquisa foi desenvolvida em consonância com os aspectos exigidos pelas legislações que regulamentam a pesquisa com seres humanos no Brasil. O estudo deriva de um macroprojeto 


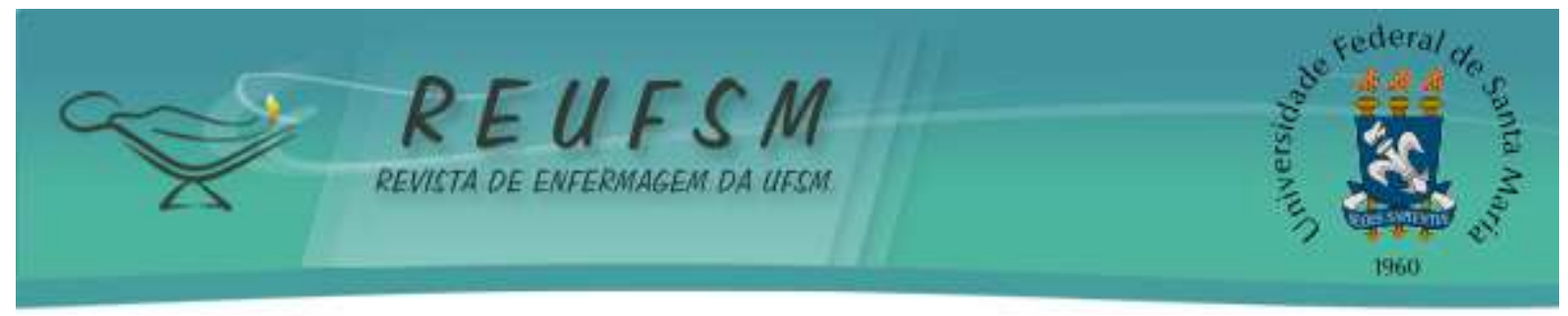

aprovado em 12/11/2012 pelo Comitê de Ética de (CAAE: 09885612.1.0000.0121) e os participantes assinaram o Termo de Consentimento Livre e Esclarecido.

\section{RESULTADOS}

Dos 27 enfermeiros, 17 (62,9\%) eram de unidades de clínica médica e 15 (55,5\%) de unidades de clínica cirúrgica. Desse total, a maioria era do sexo feminino, 25 (92,6\%); com um vínculo empregatício, 25 (92,6\%); com até cinco anos de formação profissional, 10 (37\%); e, atuantes no setor há menos de cinco anos, 15 (55,6\%). A mediana de idade foi de 32 anos (média: 35,9 anos, desvio padrão: 10), com mínima de 23 e máxima de 61. Quanto à titulação, 26 (96,3\%) enfermeiros eram pós-graduados, sendo $16(59,3 \%)$ especialistas e $10(37,0)$ mestres.

A partir do B-NWI-R, os enfermeiros avaliaram ter autonomia, boas relações com os médicos e suporte organizacional para a prática de enfermagem. No entanto, consideraram o controle sobre o ambiente uma característica desfavorável no cenário investigado. Dos 15 itens do B-NWI-R, seis apresentaram médias desfavoráveis: A, F, G, H, K e L, sendo cinco relacionados ao controle sobre o ambiente. A média obtida por cada uma das características do ambiente de trabalho dos enfermeiros está apresentada na Tabela 1.

Tabela 1 - Características do ambiente de trabalho (n=27). Florianópolis, SC, Brasil, 2012-2013.

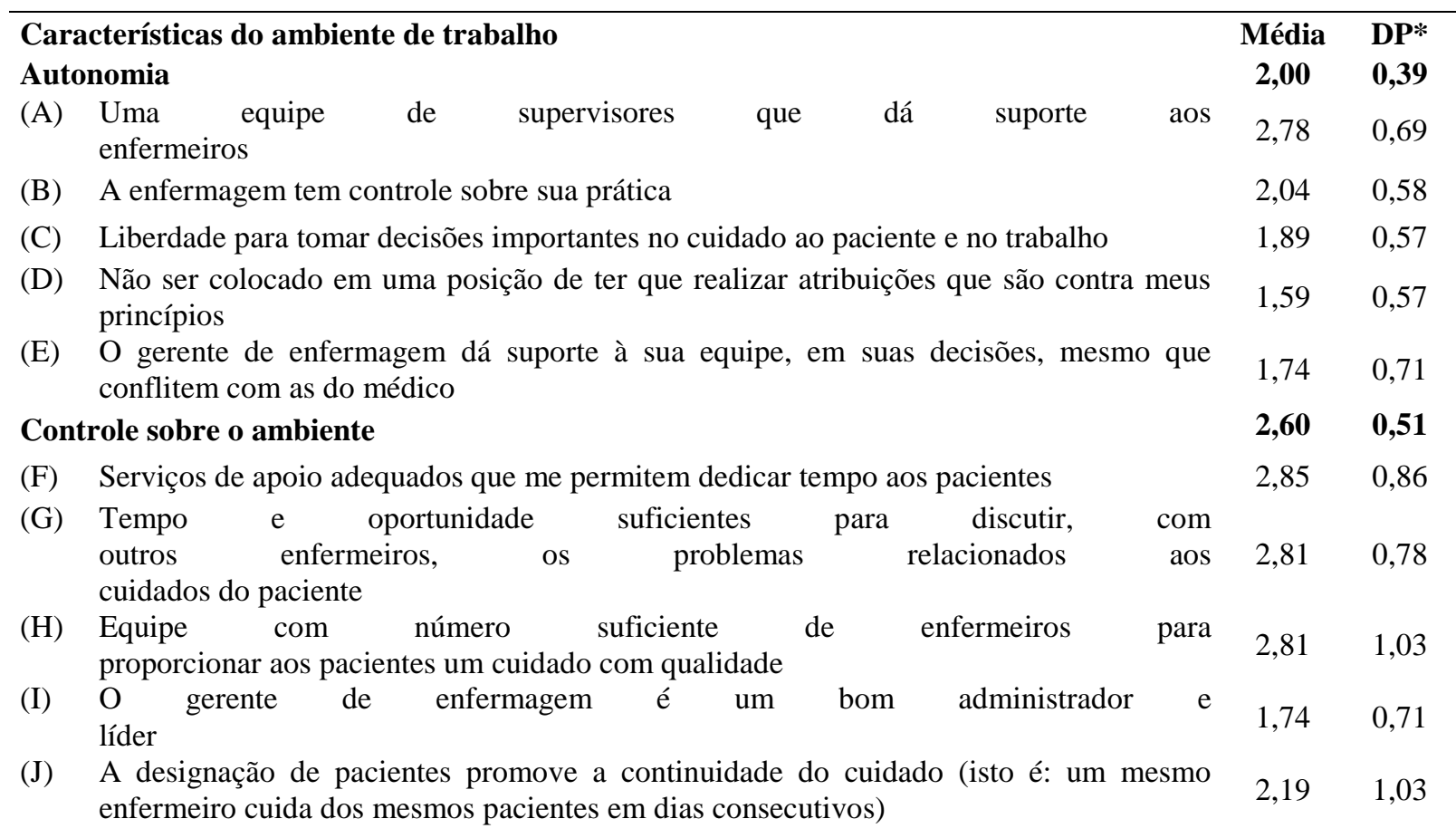




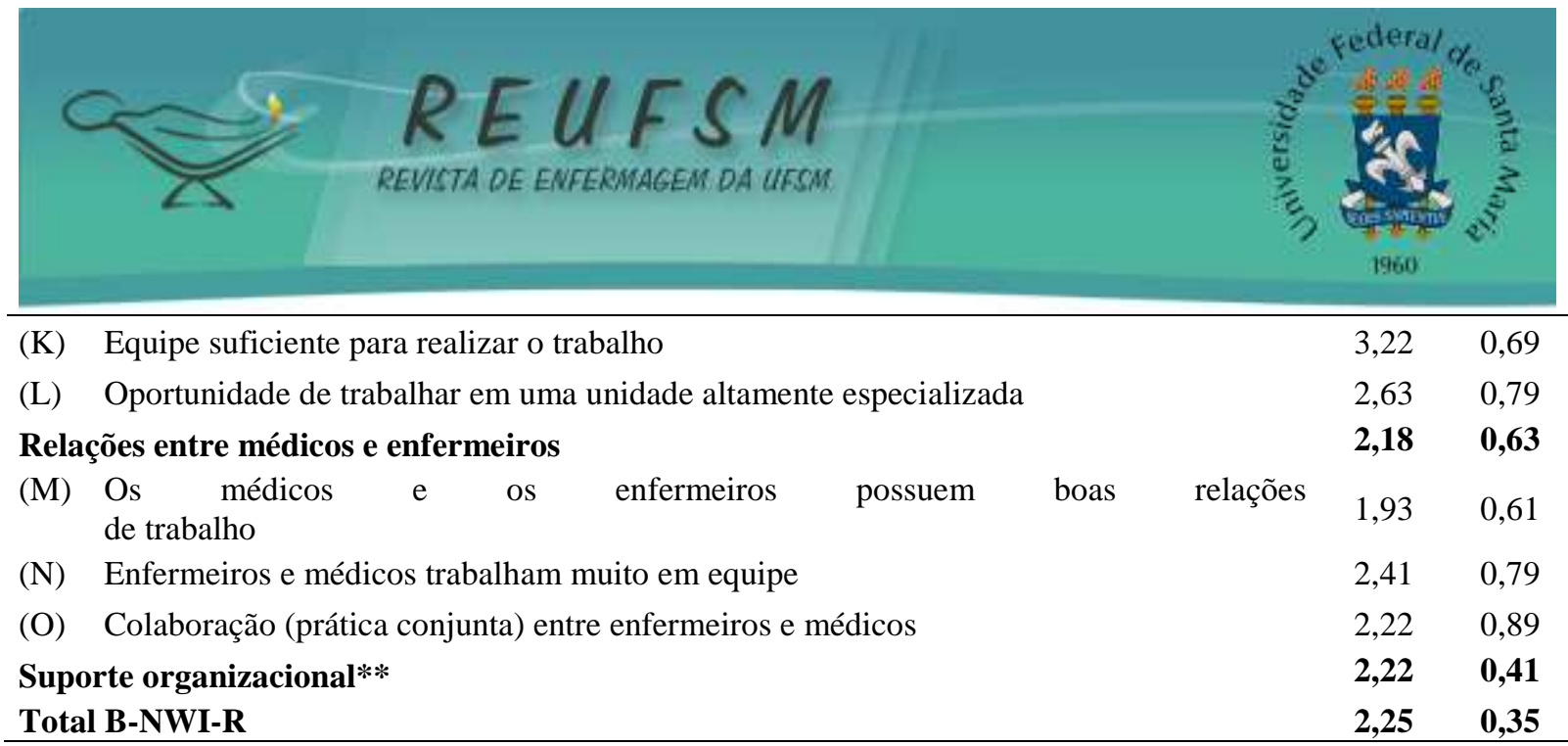

*DP = Desvio Padrão

**10 itens: B, C, D, F, G, H, I, J, M e O.

A seguir, apresentam-se os resultados qualitativos, conforme cada uma das subescalas do B-NWI-R.

\section{Autonomia}

A autonomia dos enfermeiros deriva do conhecimento acerca do que está acontecendo nas unidades de internação, tanto no que diz respeito ao contexto organizacional quanto às condições clínicas dos pacientes. Entre os aspectos que facilitam a autonomia do enfermeiro, destacaram-se a permanência da enfermagem de forma ininterrupta no contexto hospitalar e do reconhecimento pelo enfermeiro da sua importância para bom o desenvolvimento do trabalho assistencial.

O enfermeiro tem bastante liberdade para fazer as coisas, em intercorrências o enfermeiro atua sem problema nenhum. (E2) $O$ pessoal aqui fica muito atrás do enfermeiro, deixando que o enfermeiro tenha autoridade [...]. Eu me vejo como uma pessoa importante para o setor (E3). A gente tem autonomia, até porque a gente está aqui todo dia e sabe o que acontece, ficamos 24 horas com o paciente [...]. (E11)

Os enfermeiros pontuaram, ainda, a importância da experiência profissional para uma prática autônoma. A autonomia é adquirida por meio da experiência ao longo do tempo, sendo um reflexo do desenvolvimento profissional, conhecimento científico e comprometimento demonstrado pelos enfermeiros no cotidiano do trabalho.

É um espelho do que tu transpareces no teu trabalho, do teu compromisso, da tua responsabilidade [...] por meio do conhecimento $e$ da tua experiência. (E5) Então a gente tem essa autonomia, desde que claro tu tenha o teu conhecimento. (E6) [...] no começo foi muito difícil, achava que ninguém iria me respeitar, com o passar do tempo tu vai pegando mais experiência e confiança. (E11) 


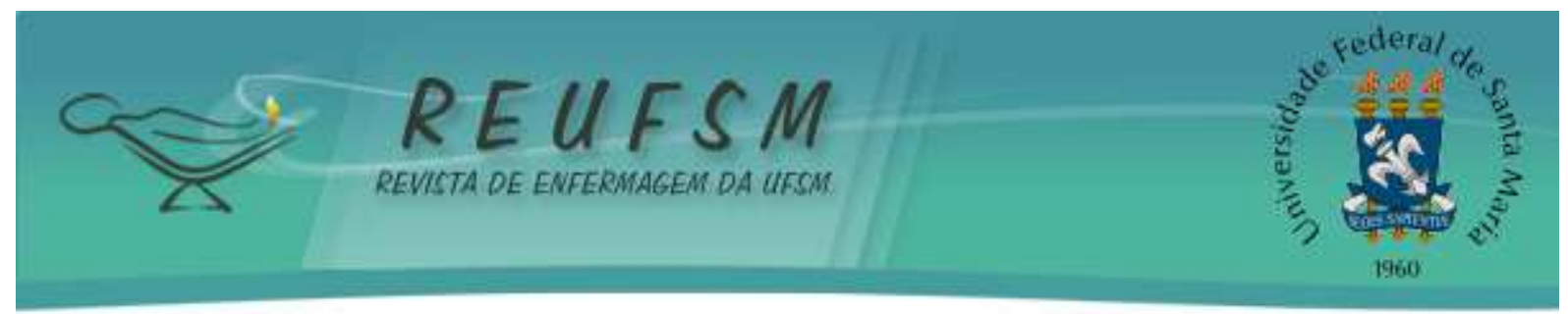

\section{Controle sobre o ambiente}

O controle do ambiente advém da centralidade do enfermeiro no contexto da organização do trabalho nas unidades de clínica médica e cirúrgica. Ele é referência para a equipe de saúde e responsável pelo gerenciamento de vários aspectos da prática assistencial, como, por exemplo, problemas no transporte de pacientes para exames e auxílio à equipe de enfermagem no desenvolvimento de atividades assistenciais.

Mesmo sendo da assistência, tu tens que resolver aqueles problemas relacionados à prática, como solucionar problemas de transporte, fazer medicação, ajudar no banho [...]. (E2) Às vezes, tenho pouco tempo e tenho que elencar prioridades, fazer os encaminhamentos junto às equipes. (E3) A enfermeira é basicamente o centro da equipe, então, o médico recorre à enfermeira e o técnico de enfermagem também recorre à enfermeira. (E4)

Como fatores que dificultam o controle sobre o ambiente, os participantes apontaram a sobrecarga de trabalho, os índices de absenteísmo e os atestados médicos de longa duração. Tais dificuldades comprometem a realização do trabalho da enfermagem, principalmente no tangente à cobertura das escalas de serviço, conforme estabelecido para o dimensionamento de cada setor.

[...] muitas vezes os técnicos, enfermeiros e médicos também têm a sua sobrecarga de trabalho. (E5) [...] as pessoas estão tendo que faltar serviço, então, ficou muito apertado. (E7) [...] nós estamos com poucos técnicos de enfermagem por causa do excesso de atestados, tu já está trabalhando a mais [...] todos esses atestados de longa duração, que às vezes são pessoas que estão fora há mais de um mês. (E8)

Um enfermeiro também destacou a demora e falta de suporte dos serviços de apoio da instituição, o que compromete a condução do trabalho e resolução de problemas relacionados à assistência.

[...] tu acaba levando sozinho, querendo conduzir os problemas, mas acaba sem conseguir resolver, até problemas de manutenção que a gente tem, aqui é tudo muito devagar, fica tudo pra enfermagem [...]. (E3)

\section{Relações entre médicos e enfermeiros}

$\mathrm{Na}$ relação com os médicos, predominou entre os enfermeiros a percepção de que existe um reconhecimento da equipe médica em relação ao trabalho desenvolvido pela enfermagem no contexto investigado. Também ressaltaram a receptividade e a abertura para o diálogo com a equipe médica. 


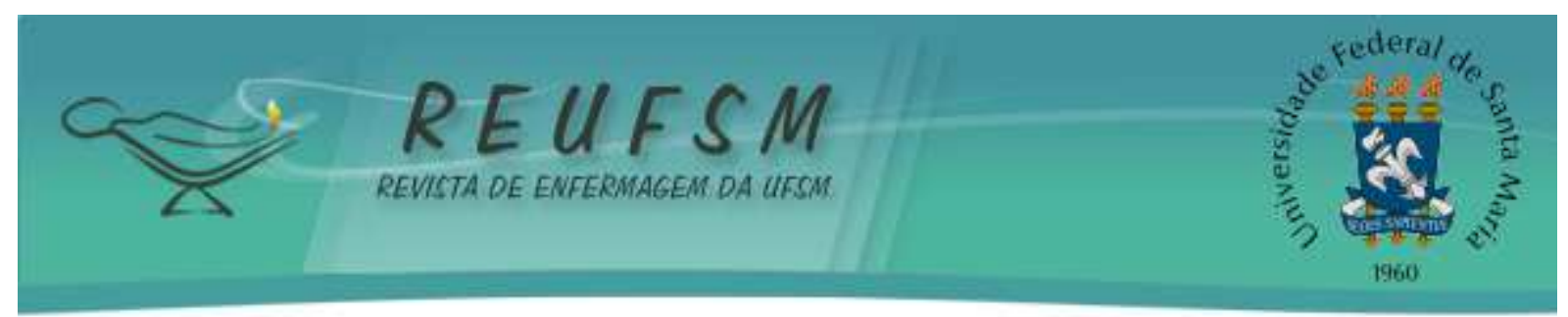

As enfermeiras aqui elas tem uma postura legal diante dos médicos, discutem os casos com eles [...]. (E2) A gente tem bastante liberdade para colocar nossa opinião, eles consideram bastante o que a gente fala. [...] a equipe médica, nutrição, psicologia e enfermagem tem uma boa relação, normalmente pacientes que necessitam de algum apoio alguma discussão, a gente sempre discute. (E10) Tu acaba tendo um diálogo. Alguns não são tão receptivos, mas normalmente é bem discutido, eles dão importância também para o que a gente coloca. (E12)

Entretanto, alguns enfermeiros comentaram sobre dificuldades no compartilhamento de decisões e condutas clínicas com os médicos. O principal aspecto de disputa na relação com os médicos tem sido a prescrição de curativos, pois nem sempre há convergência entre essas categorias profissionais quanto ao tipo de produto a ser utilizado no tratamento tópico de feridas.

[...] ainda tem muito disso, do médico achar que é ele que decide [...] tem uns que não gostam nem de respeitar a decisão do paciente [...]. (E6) Curativo é uma coisa que eu vejo muito da enfermagem e nós estamos perdendo isso aqui [...]. Eu vejo que tem curativos prescritos na prescrição médica conforme nós aprendemos na graduação e coloco na prescrição de enfermagem algo diferente [...] às vezes, nós falamos para o médico: 'eu acho que seria melhor ser essa [cobertura] daqui', e encontramos resistência. (E2) Estamos lutando para melhorar essa questão dos curativos. Tem uma polêmica bem grande entre o pensamento e a maneira de se fazer o curativo da medicina e dos enfermeiros [...]. (E13)

\section{Suporte organizacional}

O suporte organizacional foi destacado pelos enfermeiros como um aspecto positivo, principalmente em função da postura gerencial da chefia de enfermagem. A realização de reuniões mensais entre a chefia e os enfermeiros do setor permite um processo decisório compartilhado visando à discussão de problemas dos setores.

A chefia sempre toma decisões de forma compartilhada, ela sempre busca a nossa opinião. (E1) [...] é uma relação muito boa com a chefia, é uma relação bem tranquila, aberta, livre e você pode se expressar. [...] em especial ela muitas vezes não decide nada sozinha. [...] a gente faz reunião dos enfermeiros mensalmente. (E7) [...] a gente faz a reunião mensal do setor e ali então ela faz uma compilação de todas as informações, a gente discute os problemas ou traz informações. (E10) 


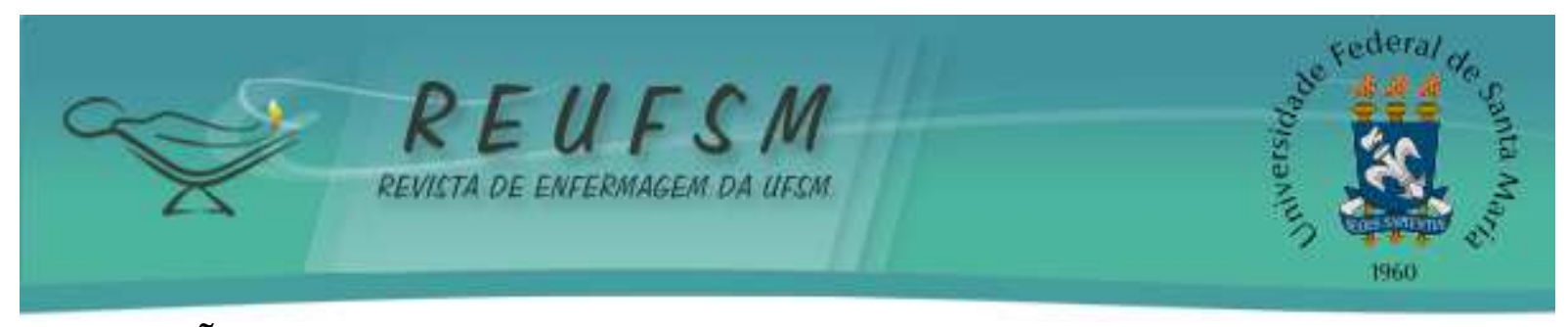

\section{DISCUSSÃO}

Este é o primeiro estudo realizado no Brasil a partir da aplicação do B-NWI-R para analisar especificamente o ambiente de trabalho de enfermeiros de unidades de clínica médica-cirúrgica. Além disso, a pesquisa também inova ao adotar uma metodologia mista no intuito de alcançar uma compreensão mais aprofundada da multiplicidade de aspectos relacionados ao ambiente de trabalho do enfermeiro no contexto hospitalar.

A partir da integração entre os resultados quantitativos e qualitativos, pode-se afirmar que os enfermeiros desenvolvem uma prática autônoma, têm um bom relacionamento com os médicos e contam com o apoio das chefias de enfermagem para o desenvolvimento do trabalho nas unidades de clínica médica e cirúrgica. No entanto, o controle sobre o ambiente é prejudicado pela falta de pessoal de enfermagem e suporte dos serviços de apoio.

Em comparação a outros estudos que utilizaram o B-NWI-R no Brasil, destaca-se que os escores encontrados nesta pesquisa $(2,25 \pm 0,35)$ foram superiores aos evidenciados em Unidades de Terapia Intensiva para pacientes adultos $(1,95 \pm 0,40)^{8}$ e em um hospital privado pediátrico $(2,08 \pm 0,58),{ }^{14}$ o que indica um ambiente de trabalho mais desfavorável nas unidades de clínica médica e cirúrgica. Além disso, a média da subescala controle sobre o ambiente obtida, mostrou-se desfavorável à prática profissional dos enfermeiros, o que difere dos resultados de estudos anteriores nos quais todas as subescalas do B-NWI-R foram positivas. ${ }^{7-9,14}$

O perfil dos atendimentos das unidades de clínica médica e cirúrgica podem explicar esses resultados. Tais setores caracterizam-se por uma rotatividade maior de pacientes, principalmente as unidades de clínica cirúrgica, as quais possuem diversas especialidades, o que aumenta a circulação de profissionais de saúde, o volume de atividades assistenciais e a demanda de recursos materiais e pessoal de enfermagem. Destaca-se também a dependência de outros setores, tais como o Centro Cirúrgico e a Unidade de Terapia Intensiva, em cirurgias mais complexas.

Especificamente em relação à clínica médica, o grau de dependência dos pacientes é maior, o que acarreta sobrecarga de trabalho para a equipe de enfermagem, principalmente pelo número reduzido de pessoal relacionado ao absenteísmo. Como consequência, os enfermeiros podem encontrar dificuldades no acompanhamento das atividades desenvolvidas e na obtenção do controle sobre o ambiente de cuidado. Esse resultado também pode ter sido influenciado pela crise nos hospitais públicos universitários no país, que tem interferido negativamente nas condições de trabalho dos profissionais de saúde. 


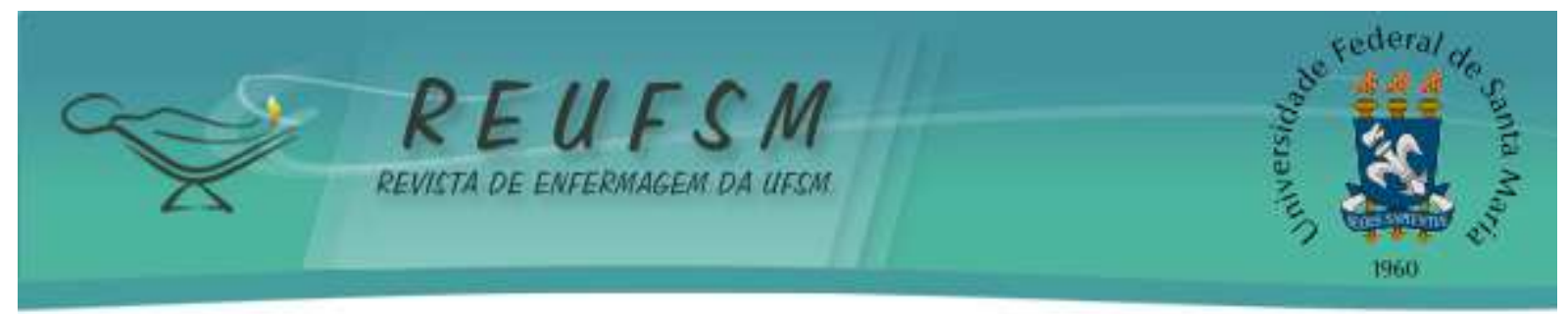

Uma das principais dificuldades dos enfermeiros em relação ao controle sobre o ambiente foi o número insuficiente de profissionais nas equipes de trabalho, principalmente em função do absenteísmo. O absenteísmo caracteriza-se pela falta ao trabalho por causas diversas e influencia diretamente no dimensionamento de pessoal. $\mathrm{O}$ dimensionamento da equipe de enfermagem, em tempo e quantidade de profissionais suficientes, é importante para a garantia da qualidade e segurança da assistência prestada aos pacientes, bem como a satisfação dos profissionais com o trabalho. Desse modo, a adoção de políticas de recursos humanos com foco na definição de critérios que regulamentem afastamentos, incluindo tempo de trabalho na instituição e avaliação de desempenho, podem minimizar os efeitos do absenteísmo no cálculo do dimensionamento. ${ }^{15}$

Outra dificuldade evidenciada nos resultados quantitativos e qualitativos foi a percepção dos enfermeiros quanto à falta de suporte dos serviços de apoio. O item F do BNWI-R (Serviços de apoio adequados que me permitem dedicar tempo aos pacientes) apresentou a média mais desfavorável entre os itens do instrumento. Os serviços de apoio correspondem à farmácia hospitalar, lavanderia, nutrição/dietética, higiene e limpeza, laboratório, banco de sangue, manutenção, entre outros. Esses serviços são fundamentais para que o resultado da assistência prestada ao paciente seja obtido com qualidade e efetividade.

Como parte das suas atribuições na gerência do cuidado, cabe ao enfermeiro zelar para que esses serviços cumpram com aquilo que lhes cabe para o tratamento do paciente e garantia de uma ambiência adequada nas unidades de internação. Nesse sentido, a falta de uma infra-estrutura adequada e de recursos assistenciais pode influenciar negativamente no cuidado e gerar insatisfação entre os profissionais. ${ }^{16}$

Quanto à autonomia do enfermeiro, nos resultados qualitativos, sobressaiu-se a percepção positiva dos enfermeiros quanto ao desenvolvimento de uma prática autônoma.

Além disso, a subescala autonomia do B-NWI-R obteve com média mais favorável em relação às demais. De forma semelhante, pesquisa com o objetivo de analisar as representações dos profissionais de saúde não enfermeiros do Rio de Janeiro sobre a autonomia profissional do enfermeiro constatou que a maioria dos participantes reconhece a existência de autonomia do enfermeiro, sendo a mesma oriunda da conquista do respeito perante outras pessoas. ${ }^{17}$

Estudo holandês sobre a relação entre o ambiente de trabalho da enfermagem e os resultados assistenciais percebidos pelos pacientes evidenciou a importância da autonomia 


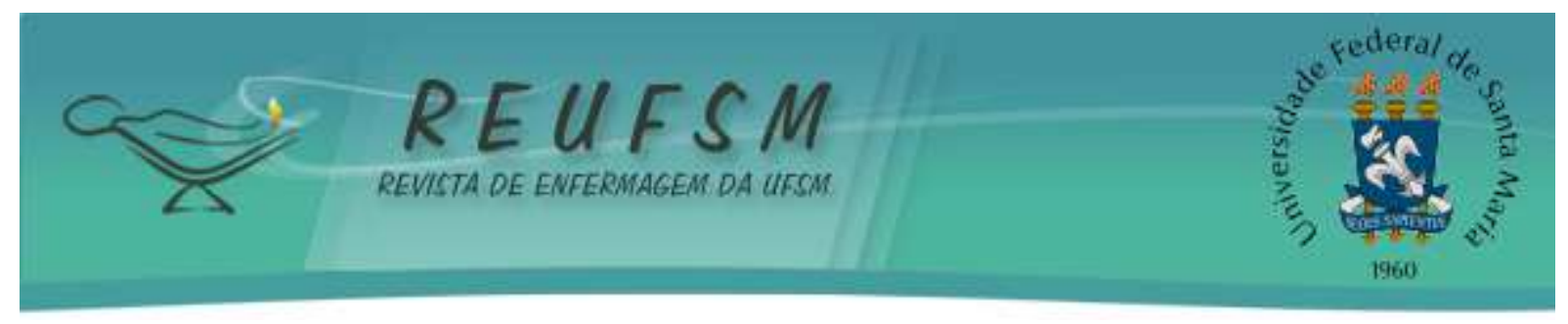

dos enfermeiros sobre sua própria prática para a qualidade dos cuidados de enfermagem prestados aos pacientes. A autonomia possibilita aos enfermeiros o controle sobre o cuidado do paciente e a tomada de decisões com base no julgamento clínico, o que repercute positivamente na satisfação assistencial dos pacientes. ${ }^{18}$

No tangente às relações entre médicos e enfermeiros, o relacionamento entre esses profissionais mostrou-se positivo nos resultados quantitativos e qualitativos do presente estudo. Nesse sentido, vale ressaltar que relações colaborativas entre os profissionais da equipe de saúde por meio da comunicação e troca de informações sobre o paciente são fundamentais para a uniformidade e efetividade do tratamento, contribuindo para a qualidade e segurança da assistência prestada aos pacientes. ${ }^{18}$

No entanto, a partir da análise dos itens $\mathrm{P}$ e $\mathrm{Q}$ do B-NWI-R, pode-se dizer que médicos e os enfermeiros possuem boas relações de trabalho, mas que nem sempre trabalham em equipe. Nos resultados qualitativos, evidenciou-se que os conflitos mais frequentes entre enfermeiros e médicos ocorrem principalmente em razão de divergências quanto à realização de curativos e práticas de cuidado com feridas.

Nesse sentido, é importante destacar que o cuidado às feridas é um campo de atuação importante para a enfermagem, o qual é regulamentado, no Brasil, pelo Conselho Federal de Enfermagem. No entanto, a falta de protocolos assistenciais nos serviços de saúde pode dificultar a realização dos cuidados de enfermagem voltados para os indivíduos portadores de feridas. Além disso, o trabalho interdisciplinar é indispensável para o estabelecimento de condutas terapêuticas eficazes a indivíduos portadores de feridas. ${ }^{19}$ Portanto, o desenvolvimento e a validação de protocolos interdisciplinares, especificando as atribuições de cada profissional, podem contribuir para a prática dos enfermeiros no cuidado de feridas no ambiente hospitalar.

Seguindo essa linha de pensamento, vale pontuar que a capacitação e o aperfeiçoamento contínuo dos enfermeiros para o cuidado de feridas também são fundamentais para o reconhecimento da sua competência para atuação nessa área. Estudo realizado na China, por exemplo, evidenciou que o desenvolvimento de um programa de capacitação sobre úlceras por pressão para profissionais de enfermagem diminuiu a ocorrência dessas lesões entre os pacientes no ambiente hospitalar, mostrando a importância da atuação da enfermagem no cuidado a lesões de pele e feriadas. ${ }^{20}$ 


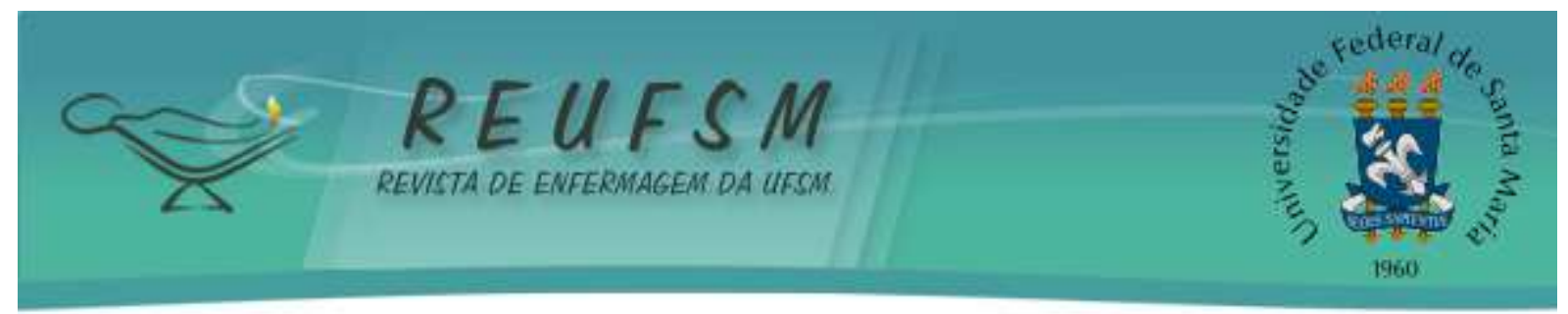

Em relação ao suporte organizacional, embora o item A do B-NWI-R (Uma equipe de supervisores que dá suporte aos enfermeiros) tenha recebido uma avaliação negativa, os resultados qualitativos reforçam evidências de que o apoio gerencial favorece o ambiente de prática profissional dos enfermeiros e contribui para a qualidade do cuidado. ${ }^{18}$ Os gestores de enfermagem tem um papel importante na promoção de um ambiente de trabalho que contribua para a satisfação profissional e retenção de enfermeiros assistenciais no ambiente hospitalar. ${ }^{21}$

Assim, por meio da sua prática gerencial, o enfermeiro pode contribuir para aprimorar a organização do trabalho e das práticas assistenciais visando à integralidade e segurança da assistência prestada. A diferença evidenciada entre resultados qualitativos e quantitativos pode estar relacionada a particularidades do processo gerencial de cada um dos setores em que os enfermeiros atuavam.

\section{CONSIDERAÇÕES FINAIS}

O ambiente de trabalho do enfermeiro em unidades de clínica médica e cirúrgica mostrou-se favorável em relação à autonomia, às relações com os médicos e enfermeiros e ao suporte organizacional. O controle sobre o ambiente foi julgado pelos enfermeiros como uma dimensão desfavorável do contexto hospitalar. Nos resultados qualitativos, destacou-se o apoio das chefias de enfermagem para o desenvolvimento do trabalho nas unidades de clínica médica e cirúrgica. As dificuldades quanto ao controle sobre o ambiente estão relacionadas à falta de pessoal de enfermagem e ao suporte dos serviços de apoio. Portanto, as características do ambiente de trabalho dos enfermeiros quanto a autonomia, controle sobre o ambiente e relações entre médicos e enfermeiros contribuem para a gerência do cuidado de enfermagem e do ambiente de trabalho pelos enfermeiros.

Dessa forma, os resultados obtidos podem contribuir para a prática profissional e pesquisa na área de enfermagem e saúde. No campo profissional, o estudo traz subsídios importantes para a gerência do cuidado de enfermagem e do ambiente de trabalho pelo enfermeiro ao evidenciar aspectos que podem melhorar o ambiente de prática de enfermeiros de unidades de clínica médica-cirúrgica e contribuir para segurança e qualidade do cuidado de enfermagem. No campo da pesquisa, esta investigação fornece subsídios para futuros estudos que avancem na discussão da problemática em voga. O estudo também pode auxiliar outros pesquisadores quanto ao uso de métodos mistos na pesquisa em enfermagem e saúde. 


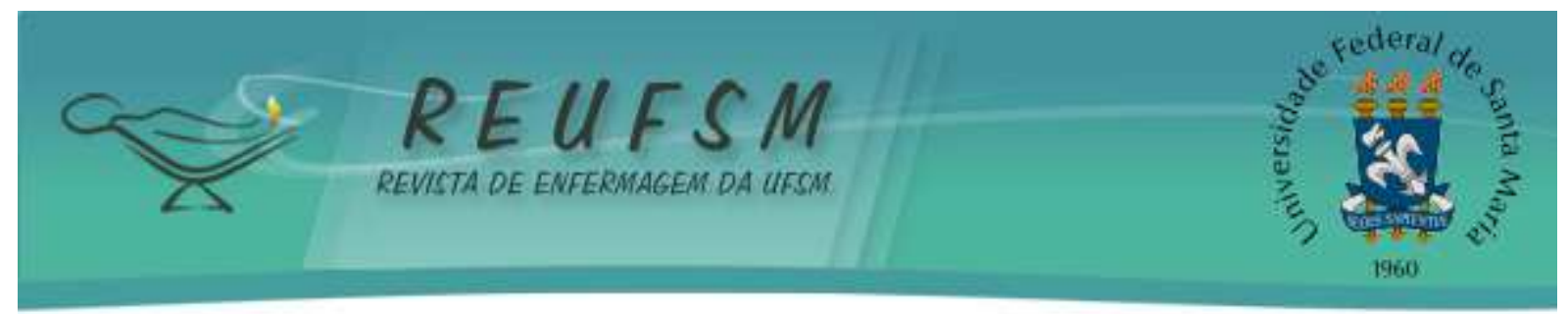

Sugere-se a realização de novos estudos sobre o presente tema em outros cenários hospitalares, incluindo a visão da equipe de enfermagem e de outros profissionais de saúde. Também é relevante investigar separadamente o ambiente de trabalho de unidades de clínica médica e cirúrgica, considerando as especificidades do cuidado de cada setor.

Entre as limitações do estudo, pontua-se a seleção dos participantes da etapa quantitativa de modo não probabilístico, pois se optou pela composição de uma amostra a partir do número total de enfermeiros dos setores onde os dados foram coletados. O estudo também foi realizado em um único hospital, sendo necessárias outras pesquisas em hospitais públicos e privados para confirmar os resultados encontrados, possibilitando comparações e possíveis generalizações.

\section{REFERÊNCIAS}

1. Brasil. Confederação Nacional de Saúde. Serviços de saúde no Brasil cadastrados no MS. [acesso em 2017 jan 13]. Disponivel em: http://www.cns.org.br/links/DADOS_DO_SETOR.htm.

2. Lorenzetti J, Gelbcke FL, Vandresen L. Management technology for hospital inpatient care units. Texto \& Contexto Enferm [Internet]. 2016 [acesso em 2017 jan 13];25(2):e1770015. Disponivel em: http://www.scielo.br/pdf/tce/v25n2/0104-0707-tce-25-02-1770015.pdf.

3. Ferreira GE, Dall'Agnol CM, Porto AR. Repercussions of proactivity in the management of care: perceptions of nurses. Esc Anna Nery [Internet]. 2016 [acesso em 2017 jan 18];20(3):e20160057. Disponível em: http://www.scielo.br/pdf/ean/v20n3/en_1414-8145-ean20-03-20160057.pdf.

4. Numminen O, Ruoppa E, Leino-Kilpi H, Isoaho H, Hupli M, Meretoja R. Practice environment and its association with professional competence and work-related factors: perception of newly graduated nurses. J Nurs Manag [Internet]. 2016 [acesso em 2017 jan 20];24(1):1-11. Disponível em: https://www.ncbi.nlm.nih.gov/pubmed/25676482.

5. Aiken L, Clarke S, Sloane D, Lake E, Cheney T. Effects of hospital care environment on patient mortality and nurse outcomes. J Nurs Adm [Internet]. 2008 [acesso em 2017 jan 21]; 38(5):223-9. Disponivel em: https://www.ncbi.nlm.nih.gov/pubmed/18469615.

6. Cucolo DF, Perroca MG. Factors involved in the delivery of nursing care. Acta Paul Enferm [Internet]. 2015 [acesso em 2017 jan 21];28(2):120-4. Disponivel em: http://www.scielo.br/pdf/ape/v28n2/1982-0194-ape-28-02-0120.pdf.

7. Oliveira PB, Spiri WC, Dell'Acqua MCQ, Mondini CCSD. Comparison between the accredited and nonaccredited public hospital working environments. Acta Paul Enferm [Internet]. 2016 [acesso em 2017 jan 21];29(1):53-9. Disponivel em: http://www.scielo.br/pdf/ape/v29n1/en_1982-0194-ape-29-01-0053.pdf.

8. Balsanelli AP, Cunha ICK. The work environment in public and private intensive care units. Acta Paul Enferm [Internet]. 2013 [acesso em 2017 jan 21];26(6):561-8. Disponível em: http://www.scielo.br/pdf/ape/v26n6/en_09.pdf. 


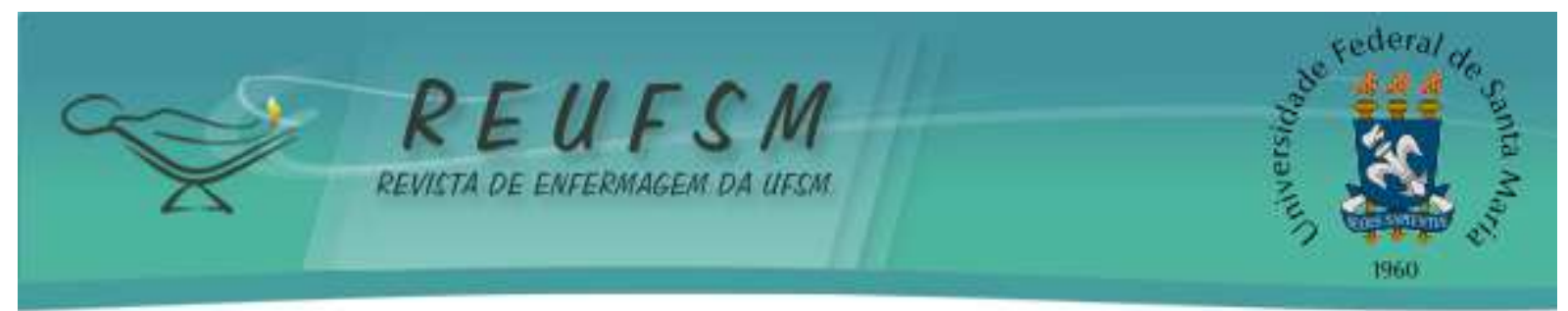

9. Panunto MR, Guirardello EB. Professional nursing practice: environment and emotional exhaustion among intensive care nurses. Rev Latinoam Enferm [Internet]. 2013 [acesso em 2017 jan 22];21(3):765-72. Disponivel em: http://www.scielo.br/pdf/rlae/v21n3/0104-1169rlae-21-03-0765.pdf.

10. Fetters MD, Curry LA, Creswell JW. Achieving integration in mixed methods designs principles and practices. Health Serv Res [Internet]. 2013 [acesso em 2017 jan 22];48(6 pt2):2134-56. Disponível em: https://www.ncbi.nlm.nih.gov/pmc/articles/PMC4097839/.

11. Gasparino RC, Guirardello EB. Translation and cross-cultural adaptation of the "Nursing Work Index" - revised. Acta Paul Enferm. [internet]. 2009 [acesso em 2017 jan 22]; 22(3):281-7. Disponivel em: http://www.scielo.br/pdf/ape/v22n3/en_a07v22n3.pdf.

12. Malterud K, Siersma VD, Guassora AD. Sample size in qualitative interview studies: guided by information power. Qual Health Res. 2015 [acesso em 2017 maio 06];26(13):175360. Disponivel em: https://www.ncbi.nlm.nih.gov/pubmed/26613970.

13. Bardin L. Análise de conteúdo. São Paulo: Edições 70; 2011. 279 p.

14. Alves DFS, Guirardello EB. Nursing work environment, patient safety and quality of care in pediatric hospital. Rev Gaúcha Enferm [Internet]. 2016 [acesso em 2017 jan 22];37(2):E58817. Disponivel em: http://www.scielo.br/pdf/rgenf/v37n2/en_0102-6933rgenf-1983-144720160258817.pdf.

15. Coelho MA, Bezerra ALQ, Fugulin FMT, Cunha CCB, Marques DO. Absenteeism in the nursing staff of clinical units of a university hospital in the center region of Brazil. Rev Urug Enferm. 2016;11(1):70-82.

16. Bugs TV, Rigo DFH, Bohrer CD, Borges F, Oliveira JLC, Tonini NS. Dificuldades do enfermeiro no gerenciamento da unidade de pronto-socorro hospitalar. Rev Enferm UFSM [Internet]. 2017 jan/fev [acesso em 2017 out 10];7(1):90-9. Disponível em: https://periodicos.ufsm.br/reufsm/article/view/23374/pdf doi: 10.5902/2179769223374.

17. Santos EI, Alves YR, Gomes AMT, Ramos RS, Cerqueira ASSS, Santo CCE. Social representations of nurses' professional autonomy among non-nursing health personnel. Rev Enferm UERJ [Internet]. 2015 [acesso em 2017 jan 26];23(4):481-7. Disponível em: http://www.objnursing.uff.br/index.php/nursing/article/view/5294/pdf_1.

18. Kieft R, Brouwer B, Francke A, Delnoij D. How nurses and their work environment affect patient experiences of the quality of care: a qualitative study. BMC Health Serv Res [Internet]. 2014 [acesso em 2017 jan 30];14:249. Disponivel em: http://bmchealthservres.biomedcentral.com/articles/10.1186/1472-6963-14-249.

19. Sehnem GD, Busanello J, Silva FM, Poll MA, Borges TAP, Rocha EM. Difficulties faced by nurses in nursing care for individuals with wounds. Cienc Cuid Saúde [Internet]. 2015 [acesso em 2017 fev 03];14(1):839-46. Disponível em: http://periodicos.uem.br/ojs/index.php/CiencCuidSaude/article/view/20949.

20. Feng $\mathrm{H}, \mathrm{Li} \mathrm{G}, \mathrm{Xu} \mathrm{C}, \mathrm{Ju} \mathrm{C}$. Educational campaign to increase knowledge of pressure ulcers. Br J Nurs [Internet]. 2016 [acesso em 2017 fev 03];25(12):30-5. Disponível em: https://www.ncbi.nlm.nih.gov/pubmed/27345080. 


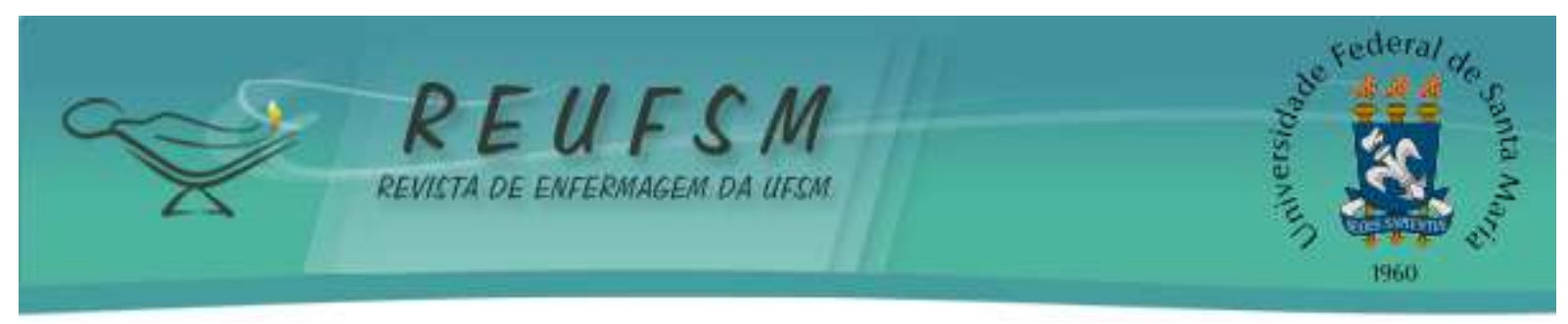

21. Lartey S, Cummings G, Profetto-McGrath J. Interventions that promote retention of experienced registered nurses in health care settings: a systematic review. J Nurs Manag [Internet]. 2014 [acesso em 2017 fev 03];22(8):1027-41. Disponível em: https://www.ncbi.nlm.nih.gov/pubmed/23758834.

Data de submissão: 10/10/2017

Data de aceite: $12 / 03 / 2018$

Autor correspondente: José Luís Guedes dos Santos

Email: joseenfermagem@gmail.com

Endereço: Campus Reitor João David Ferreira Lima, s/n - Trindade, Florianópolis - SC

CEP: 88040970 